\title{
Neuroprotective effects of magnesium L-threonate in a hypoxic zebrafish model
}

\author{
Young-Sung Kim¹, Young Ju Won' ${ }^{1}$ Byung Gun Lim¹, Too Jae Min², Yeon-Hwa Kim³ and II Ok Lee ${ }^{1 *}$ (D)
}

\begin{abstract}
Background: Hypoxia inhibits the uptake of glutamate (a major neurotransmitter in the brain closely related to cognitive function) into brain cells, and the initial response of cells to cortical hypoxia depends on glutamate. Previous studies have suggested that magnesium may have protective effects against hypoxic injuries. In particular, magnesium L-threonate (MgT) may increase magnesium ion concentrations in the brain better than $\mathrm{MgSO}_{4}$ and improve cognitive function.

Methods: We evaluated cell viability under hypoxic conditions in the MgT- and $\mathrm{MgSO}_{4}$-treated human $\mathrm{SH}_{-} \mathrm{SY} 5 \mathrm{Y}$ neurons, in vivo behavior using the T-maze test following hypoxia in MgT-treated zebrafish, activity of brain mitochondrial dehydrogenase by 2,3,5-triphenyltetrazolium chloride (TTC) staining, and protein expression of the excitatory amino acid transporter (EAAT) 4 glutamate transporter by western blotting.

Results: Among the groups treated with hypoxia, cell viability significantly increased when pre-treated with 1 or $10 \mathrm{mM} \mathrm{MgT} \mathrm{(} p=0.009$ and 0.026, respectively). Despite hypoxic insult, MgT-treated zebrafish showed preferences for the red compartment ( $p=0.025$ for distance and $p=0.007$ for frequency of entries), suggesting memory preservation. TTC staining showed reduced cerebral infarction and preserved absorbance in the MgT-treated zebrafish brain after hypoxia ( $p=0.010$ compared to the hypoxia group). In addition, western blot showed upregulation of EAAT4 protein in the MgT treated group.
\end{abstract}

Conclusions: Pre-treatment with MgT attenuated cell death and cerebral infarction due to hypoxia and protected cognitive function in zebrafish. In addition, MgT appeared to modulate expression of the glutamate transporter, EAAT4.

Keywords: Behavior, Glutamate, Hypoxia, Magnesium, Neuroprotection, Zebrafish

\section{Background}

With a growing need to preserve cognitive function in an aging population, various drugs that can improve memory are being studied [1-3]. However, there is limited evidence of efficacy and limited indications for use of cognition-enhancing drugs including psychostimulants and glutamate activators. Misuse of such drugs may cause side effects and complications as well as social

\footnotetext{
*Correspondence: iloklee@korea.ac.kr

${ }^{1}$ Department of Anesthesiology and Pain Medicine, Korea University Guro Hospital, Seoul, Korea

Full list of author information is available at the end of the article
}

problems [3]. Therefore, it is necessary to establish more in-depth knowledge and scientific evidence to support the clinical applications of such drugs.

Hypoxic insults may result in rapid, irreversible, ischemic damage to neurons $[4,5]$, and a variety of functional deficits including cognitive impairments. Hypoxia is known to inhibit the migration of glutamate (one of the main neurotransmitters in the brain, closely related to cognitive function) into brain cells $[1,6,7]$. Previous studies confirmed that the initial response of cells to cortical hypoxia depends on glutamate [8]. Excitatory amino acid transporter 4 (EAAT4), a glutamate receptor, shows 
decreased immunoreactivity after hypoxic-ischemic damage [9].

Several studies have shown that magnesium sulfate $\left(\mathrm{MgSO}_{4}\right)$ reduces levels of reactive oxygen species and inflammation following hypoxic injuries [10]. However, the increase in magnesium ion levels in the cerebrospinal fluid (CSF) following $\mathrm{MgSO}_{4}$ administration is limited by central nervous system regulation and the bloodbrain barrier. Even in the case of a 150-200\% increase in plasma magnesium concentrations in humans, that in the CSF increases by only 10-19\% [11]. The relatively newly developed magnesium L-threonate $(\mathrm{MgT})$ consists of a magnesium ion and threonate, which exists physiologically in the brain [12]. Unlike other magnesium compounds, MgT significantly increases magnesium ion levels in the CSF [13]. Previous studies have suggested several neuroprotective mechanisms of MgT in the rat using Alzheimer's disease and neuropathy models [14-16]. Wei et al. showed that MgT prevented the reduction in glutamatergic synaptic transmission under Alzheimer's disease-like pathological conditions [16]. We expected that their findings would be similarly applicable to the hypoxic model.

To assess the effects of magnesium on cognitive function, we used a hypoxic zebrafish model. Zebrafish demonstrate a $70 \%$ genetic similarity to human protein coding genes [17] and an $84 \%$ similarity in disease-related genes [18]; moreover, the zebrafish neurotransmitter system is similar to that of mammals [19]. This model has recently become regarded as an ideal vertebrate model that has a competitive edge in terms of time and cost for large-scale drug toxicity screening studies [20]. In our previous study, zebrafish were found to be useful in the evaluation of cognitive function including learning and memory [21].

In this study, we investigated whether MgT is associated with neuroprotection and improvement in cognitive function using a hypoxic zebrafish model. We hypothesized that magnesium would prevent hypoxia-induced cognitive dysfunction, decrease infarcted brain area, and upregulate the glutamate transporter, EAAT4. Further, we predicted that glutamate would have a role in the mechanism underlying MgT-induced neuroprotection.

\section{Methods}

\section{Experimental animal}

Adult zebrafish (4-6 months old, $2.5-3.5 \mathrm{~cm}$ long, $350 \pm 50 \mathrm{mg}$ of weight, wild type, Danio rerio) were used in the study. Animals were fed brine shrimp twice a day in a $28.5^{\circ} \mathrm{C}$ tank, and were on a 14-h daytime and 10 -h night cycle. The watercraft was equipped with a multi-stage filtration system with a sediment filter, post-carbon filter, fluorescent UV light, and sterilization filter (Zebrafish
AutoSystem, Genomic Design, Daejeon, Korea). All procedures were reviewed and approved by the Committee on the Ethics of Animal Experiments of the Korea University Medical School (IACUC number KOREA-20180032). After behavior experiments, all zebrafish were anesthetized using MS-222 (tricaine, Sigma-Aldrich, St. Louis, Mo., USA) and euthanatized by decapitation.

\section{Drug administration}

$\mathrm{MgT}\left(\mathrm{C}_{8} \mathrm{H}_{14} \mathrm{MgO}_{10}\right.$, molecular weight $294.50 \mathrm{~g} / \mathrm{mol}$, Doctor's Best Inc., USA) and $\mathrm{MgSO}_{4}$ (anhydrous, molecular weight $120.37 \mathrm{~g} / \mathrm{mol}$, Biosesang Inc., Korea) were used in the study. MgT were centrifuged at 2000 revolutions per minute for $2 \mathrm{~min}$ in $30 \mathrm{~mL}$ of phosphate-buffered saline (PBS) to a concentration of $100 \mathrm{mM}$ and a sample from the middle layer was diluted 10 times $(10 \mathrm{mM})$ or 100 times $(1 \mathrm{mM}) . \mathrm{MgSO}_{4}$ was also centrifuged and diluted in PBS in the same way. Drug concentrations were determined based on physiological concentrations of magnesium [22, 23] and results of MgT toxicity experiments in zebrafish embryos described below.

\section{Evaluation of toxicity of MgT in zebrafish embryos}

Zebrafish embryos were treated with 0 (control), 5, 25, 50 , and $100 \mathrm{mM} \mathrm{MgT}$ (diluted in water) in a 6-well plate (ten embryos per well) and incubated at $26{ }^{\circ} \mathrm{C}$. Two days later, heart rate and development were measured with a microscope. After 6 days, survival rate was evaluated.

\section{Hypoxic chamber}

The hypoxic chamber consisted of a closed glass box attached to an inner lid with a pack of Gaspak ${ }^{\mathrm{TM}}$ (Becton, Dickinson and Company, USA) (Fig. 1) filled with water such that the Gaspak ${ }^{\mathrm{TM}}$ was not immersed. The hypoxic chamber was closed the night prior to the experiment so as to reach at $1.0 \pm 0.5 \mathrm{mg} / \mathrm{L}$ of dissolved oxygen (DO) as measured by a portable $\mathrm{DO}$ analyzer.

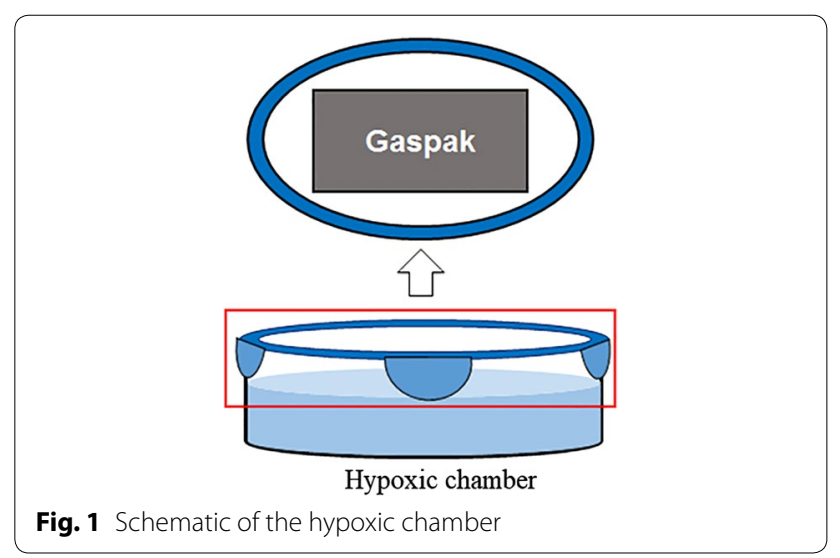




\section{Effects of $\mathrm{MgT}$ and $\mathrm{MgSO}_{4}$ on neurons}

The human neuroblastoma cell line SH-SY5Y $\left(\right.$ ATCC $^{\circledR}$ CRL-2266 ${ }^{\mathrm{TM}}$, American Type Culture Collection, USA) were used for in vitro experiments. After ensuring cells were healthy and free of contamination, and removing culture media, cells were treated with fetal bovine serum-free Dulbecco's Modified Eagle Medium, and starved for two hours. Prewarmed $1 \times$ trypsin solution was added to promote cell detachment in appropriate quantities $\left(0.5 \mathrm{~mL} / 10 \mathrm{~cm}^{2}\right)$. When more than $90 \%$ of cells were detached, prewarmed complete growth media was added to inactivate the trypsin. After cell counting, $\mu \mathrm{L}$ of cell suspension was added to a 96-well plate to obtain a final concentration of 7000 cells per well. The cells were treated with $\mathrm{PBS}$ (control), $\mathrm{MgSO}_{4}$ (1 or $10 \mathrm{mM}$ ) or $\mathrm{MgT}$ ( 1 or $10 \mathrm{mM}$ ) for $1 \mathrm{~h}$. Media was changed, and the cells were incubated for two hours in a hypoxic chamber with no water (or a regular $\mathrm{CO}_{2}$ incubator as a control). To evaluate cell viability, $10 \mu \mathrm{L}$ of cell counting kit- 8 solution (CCK- 8 solution, Dojindo Laboratories, Japan) was added to each well and absorbance was measured with a microplate reader $(450 \mathrm{~nm})$. To ensure reliability of the experimental results, experiments were repeated three times (each with 6 technical replicates per group).

\section{Hypoxic zebrafish model}

Zebrafish exposed to hypoxia were evaluated using a 4-stage behavioral repertoire as previously described [24]. Briefly, fish were classified as stage 1 (swimming on the surface of the water), stage 2 (failure to attain normal posture), stage 3 (intermittent maintenance of opercular beats with brief movements), and stage 4 (dead). When a hypoxic zebrafish reached stage 3 it was immediately transferred to a normoxic chamber (DO: $7.0 \pm 0.5 \mathrm{mg} / \mathrm{L}$ ).

\section{Oral administration of PBS and MgT}

Oral administration was performed by a professional after tricaine anesthesia [25]. Zebrafish were anesthetized in a water mixture containing $16.8 \mathrm{mg}$ of tricaine per $100 \mathrm{~mL}$, resuspended to minimize the anesthetic time, and administered $1 \mu \mathrm{L}$ orally of PBS or MgT $(10 \mathrm{mM})$ with a micropipette, taking care not to damage the mouth. This volume was chosen based on pilot data showing that zebrafish were likely to vomit solutions administered at volumes greater than $2 \mu \mathrm{L}$.

\section{Classification of the experimental group}

The experimental group was randomly divided into four groups: $\mathrm{PBS}=$ zebrafish that were orally administered $1 \mu \mathrm{L}$ of PBS and remained in normoxia for $3 \mathrm{~h}$; $\mathrm{PBS}+\mathrm{HYP}=$ zebrafish that were orally administered $1 \mu \mathrm{L}$ of PBS and placed in the hypoxic chamber for $1 \mathrm{~h}$ followed by normoxia for $2 \mathrm{~h} ; \mathrm{MgT}=$ zebrafish that were orally administered $10 \mathrm{mM}$ of $\mathrm{MgT}$ and were in normoxia for $3 \mathrm{~h}$; and the MgT + HYP group=zebrafish that were orally administered $10 \mathrm{mM}$ of $\mathrm{MgT}$ and placed in the hypoxic chamber for $2 \mathrm{~h}$ followed by normoxia for $2 \mathrm{~h}$ (Fig. 2a). Randomization was performed using a

\begin{tabular}{|c|c|c|c|c|c|}
\hline Groups & Day $1 \sim 4$ & & & Day 5 & \\
\hline PBS & \multirow{4}{*}{$\begin{array}{l}\text { Training } \\
\text { period }\end{array}$} & \multirow{2}{*}{$\begin{array}{c}\text { Oral PBS } \\
\text { administration }\end{array}$} & \multicolumn{2}{|c|}{ Normoxia for 3 hour } & \multirow{4}{*}{$\begin{array}{c}\text { T-maze } \\
\text { test }\end{array}$} \\
\hline $\mathrm{PBS}+\mathrm{HYP}$ & & & $\begin{array}{l}\text { Normoxia } \\
\text { for } 1 \text { hour }\end{array}$ & $\begin{array}{l}\text { Hypoxic treatment and return } \\
\text { to normoxia for } 2 \text { hours } \\
\end{array}$ & \\
\hline $\mathrm{Mg} \mathrm{T}$ & & \multirow{2}{*}{$\begin{array}{c}\text { Oral MgT } \\
\text { administration }\end{array}$} & \multicolumn{2}{|c|}{ Normoxia for 3 hour } & \\
\hline $\mathrm{MgT}+\mathrm{HYP}$ & & & $\begin{array}{l}\text { Normoxia } \\
\text { for } 1 \text { hour }\end{array}$ & $\begin{array}{l}\text { Hypoxic treatment and return } \\
\text { to normoxia for } 2 \text { hours }\end{array}$ & \\
\hline
\end{tabular}

b

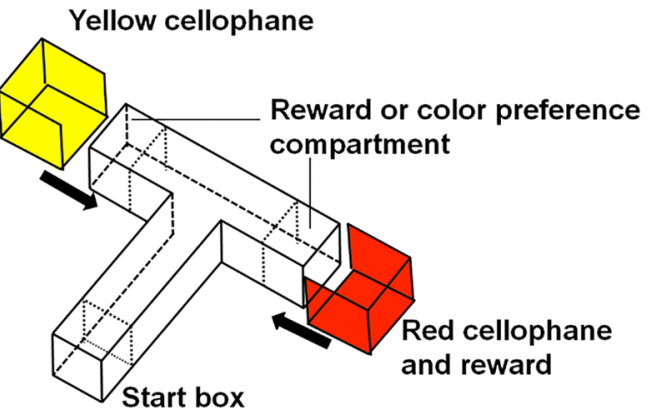

Fig. 2 T-maze experiment. a Experimental groups and schedules. b Three-dimensional schematic of the T-maze. Colors indicate the two compartments: red for the target compartment and yellow for the opposite compartment 
web-based computer-generated list (www.randomizat ion.com). The numbers were kept in opaque, sealed envelopes that were opened in the laboratory just before the experiment.

\section{Sample size calculation}

Power analysis revealed that a minimum sample size of 10 for each group would be required to achieve a power of $80 \%$ at a significance level of $5 \%$. Power analysis was calculated from results of our prior experiment [26]. To allow for exclusions, total sample size was prospectively set at 48 zebrafish (12 for each group).

\section{Color-added T-maze test}

A T-shaped maze containing two arms and one stem was used to measure learning using color preference or compensation. A starting box $(10 \times 10 \times 10 \mathrm{~cm})$ was located at the bottom of the stem $(50 \times 10 \times 10 \mathrm{~cm})$ and two compartments (the "target" and "opposite" compartment; $10 \times 10 \times 10 \mathrm{~cm}$ each) were located at the ends of the maze arms (each $20 \times 10 \times 10 \mathrm{~cm})$. Transparent sliding doors were used to separate the start box and the arms of the maze from the stem. During the training periods, sleeves made of red or yellow cellophane were fitted around the target and opposite compartments, respectively (Fig. 2b). To minimize bias, all the experiments started at 1-2 pm in a quiet dedicated place in the laboratory and all zebrafish were subjected to a habituation trial for $2 \mathrm{~h}$ prior to testing. Each zebrafish was trained once a day for four consecutive days. $20 \mu \mathrm{L}$ of food (brine shrimp) was placed in the red cellophane compartment and each zebrafish was placed in the start box. On the fifth day, all zebrafish underwent memory testing, wherein all cellophane and food rewards were removed from the maze. All processes were recorded with a $4 \mathrm{~K}$ camcorder (Sony FDR-AX33, Sony Corporation, Japan) and analyzed using EthoVision XT software (Noldus Information Technology, Netherlands).

\section{Evaluation of MgT effects on zebrafish behavior}

The evaluation indices for zebrafish behavior were as follows: time spent $=$ total time spent in one of the compartments of the T-maze; distance moved=total horizontal distance moved in a compartment; and frequency of entries $=$ the number of times the zebrafish entered one of the compartments.

Compartment preference was calculated using the following equation: preference $=\log$ (target compartment/opposite compartment). Therefore, 0 indicated no preference, and 1 indicated an index value in the target compartment 10 times higher than that of the opposite compartment. Conversely, a negative preference value indicated that the opposite compartment was preferred.

\section{2,3,5-triphenyltetrazolium chloride (TTC) staining}

TTC staining is a widely used method to measure hypoxic brain damage [27] and evaluate activity of brain mitochondrial dehydrogenase. To remove the brain, zebrafish were anesthetized using MS-222 (tricaine) and euthanatized by decapitation upon completion of behavior testing. For gross infarct size assessment, the brain was incubated in $1 \mathrm{~mL}$ of PBS containing 2\% TTC (Sigma-Aldrich) for $40 \mathrm{~min}$, followed by overnight incubation in $4 \%$ paraformaldehyde. The following day, the brain was imaged with a microscope. For absorbance measurement, the brain was incubated in TTC solution in a $\mathrm{CO}_{2}$ incubator for $100 \mathrm{~min}$. The TTC solution was discarded and the brain was gently rinsed with 2-3 drops of dimethyl sulfoxide (DMSO)/ethanol (1:1 solution) and stored overnight in a $1.5 \mathrm{~mL}$ tube containing $1 \mathrm{~mL}$ of DMSO/ethanol solution. The following day, the absorbance of the DMSO/ethanol solution was measured with a spectrophotometer (Epoch, BioTek instruments, USA) and corrected by the brain weight.

\section{Western blot}

To determine the mechanism of MgT in neuroprotection during hypoxia, we measured the expression of a glutamate transporter, excitatory amino acid transporter (EAAT) 4, by western blot. Following behavioral testing, zebrafish brains were homogenized in a lysis buffer (radio-immunoprecipitation buffer; Sigma) containing a protease inhibitor cocktail (Roche). Protein concentration was determined using the Bradford method. Proteins $(20 \mu \mathrm{g})$ were separated by $8 \%$ sodium dodecyl sulfate polyacrylamide gel electrophoresis and transferred to membranes. The membranes were blocked with 5\% skim milk in $1 \times$ tris-buffered saline (TBS) at room temperature for 1 hour. The membranes were then incubated with a rabbit anti-EAAT4 antibody (ab41650, Abcam, Cambridge, MA) or rabbit anti-beta actin (A5441, SigmaAldrich) overnight at $4{ }^{\circ} \mathrm{C}$. Membranes were washed three times in $1 \times \mathrm{TBS}+0.05 \%$ Tween 20 and incubated with a 1:2000 (EAAT4) or 1:5000 (beta actin) dilution of horseradish peroxidase (HRP)-conjugated anti-rabbit immunoglobulin G (IgG) secondary antibody. An electro-chemiluminescence kit was used to develop the western blots (Amersham, Boston, MA, USA). Quantitative analysis of densitometry was performed using ImageJ (v. 1.52a, National Institutes of Health, USA).

\section{Statistical analysis}

All data were analyzed using SPSS 22 (IBM, USA) and GraphPad Prism 6.0 (GraphPad, USA). Data are expressed as mean with standard error of the mean. Data were tested for normality using the KolmogorovSmirnov test. Parametric or non-parametric analysis was 
performed as appropriate. For MgT toxicity and survival data, a Kaplan-Meier survival curve was evaluated using the log-rank (Mantel-Cox) test. Cell viability was compared using one-way analyses of variance, and Tukey's multiple comparison tests were used for post hoc analysis among the treated and untreated hypoxia groups. T-maze results were compared using a paired $\mathrm{T}$ test or Wilcoxon matched-pair signed rank test to determine differences in compartment preference. P-value $<0.05$ was considered significant.

\section{Results}

\section{Evaluation of toxicity of MgT in zebrafish embryos}

Two embryos in the $100 \mathrm{mM}$ group died on the day of MgT treatment. Two days after drug treatment, most zebrafish in the $100 \mathrm{mM}$ group showed abnormal cardiac development. The remaining embryos that survived past the third day hatched. On the sixth day, all zebrafish in the control, $\mathrm{MgT}$ (5 mM), and $\mathrm{MgT}(25 \mathrm{mM}$ ) groups survived (10 zebrafish in each group, survival rate of 100\%). Two survived in the $\mathrm{MgT}(50 \mathrm{mM})$ group (survival rate of $20 \%)$ and no zebrafish survived in $\mathrm{MgT}(100 \mathrm{mM})$ group (survival rate of $0 \%$ ) Kaplan-Meier survival analysis showed significant differences between doses $(\mathrm{p}<0.001)$ (Fig. 3).

\section{Effects of $\mathrm{MgT}$ and $\mathrm{MgSO}_{4}$ on neuron cells}

In the groups that underwent hypoxia, cell viability was significantly decreased compared to the groups without hypoxic treatment. Among the normoxic groups, cell viabilities decreased when pretreated with $\mathrm{MgT}$ or $\mathrm{MgSO}_{4}$ $(\mathrm{F}(2,51)=84.65, \mathrm{p}<0.001$ and $\mathrm{F}(2,51)=81.18, \mathrm{p}<0.001$, respectively) (Fig. 4). On the contrary, among the hypoxic groups, cell viability significantly increased when pretreated with $1 \mathrm{mM}$ or $10 \mathrm{mM} \mathrm{MgT}(\mathrm{F}(2,51)=6.837$, $\mathrm{p}=0.0023$ ) (Fig. 4). In the groups with $1 \mathrm{mM}$ or $10 \mathrm{mM}$ $\mathrm{MgSO}_{4}$, cell viability was not affected $(\mathrm{F}(2,51)=0.6304$, $\mathrm{p}=0.5365)$. In both $\mathrm{MgT}$ and $\mathrm{MgSO}_{4}$, Concentration

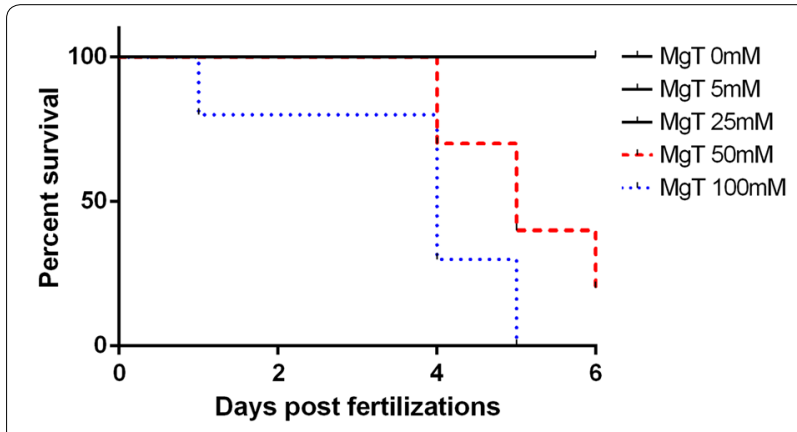

Fig. 3 Kaplan Meier Survival Analysis. The log-rank (Mantel-Cox) test showed significant differences among the survival curves $(p<0.001)$

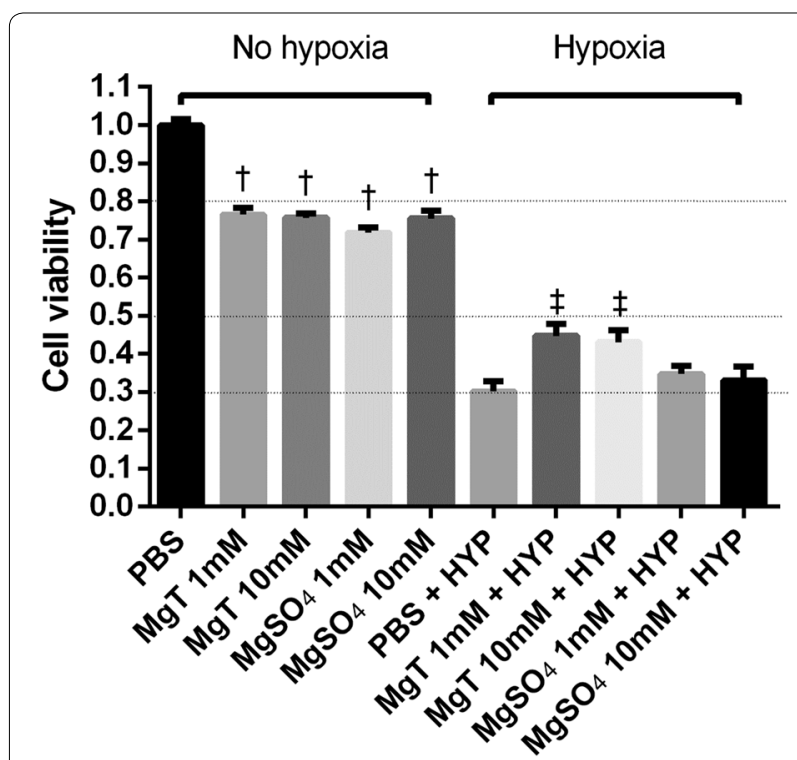

Fig. 4 Effects of $\mathrm{MgT}$ and $\mathrm{MgSO}_{4}$ on neurons after normoxia (left) or hypoxia (right) in the presence or absence of $\mathrm{MgT}_{\text {or }} \mathrm{MgSO}_{4}$. $\mathrm{MgT}$ : magnesium L-threonate. The cells were treated with PBS (control), $\mathrm{MgSO}_{4}$ (1 or $10 \mathrm{mM}$ ) or $\mathrm{MgT}$ (1 or $10 \mathrm{mM}$ ) for $1 \mathrm{~h}$. After the culture media were exchanged, the cells were incubated for $2 \mathrm{~h}$ in a $\mathrm{CO}_{2}$ incubator or a hypoxic chamber. The cellular experiments were repeated three times (each individual experiment contained 6 technical replicated, totaling 180 wells). Data are shown as mean \pm SEM. ${ }^{\dagger} p<0.05$ compared to the PBS group, ${ }^{\neq} p<0.05$ compared to the PBS + HYP group. PBS: phosphate-buffered saline; MgT, magnesium L-threonate; HYP, hypoxia

itself ( 1 or $10 \mathrm{mM})$ did not affect any significant difference, regardless of oxygen condition.

\section{Evaluation of MgT effects in Zebrafish behavior}

Twelve zebrafish in the PBS group, 11 in the PBS + HYP group, 11 in the MgT group, and 11 in the MgT + HYP group were included in the behavior testing. In the hypoxia groups, the average time for the zebrafish to reach stage 3 of hypoxia was $10 \pm 0.5 \mathrm{~min}$.

The PBS group showed significantly longer time spent and distance moved in the target compartment than the opposite compartment $(\mathrm{t}(11)=2.420, \mathrm{p}=0.034$ and $\mathrm{t}$ $(11)=2.243, p=0.046$, respectively) while the frequency of entries was not significantly different $(t(11)=1.267$, $\mathrm{p}=0.231$ ). There was no significant difference in time, distance, or frequency of entries between the target and opposite compartments in the PBS + HYP group ( $\mathrm{t}(10)=0.201, \mathrm{p}=0.845, \mathrm{t}(10)=0.011, \mathrm{p}=0.991$, and t $(10)=0.686, p=0.509$, respectively) (Table 1$)$. The $\mathrm{MgT}$ group also showed a significantly longer time and distance in the target compartment compared to the opposite compartment ( $\mathrm{t}(10)=2.901, \mathrm{p}=0.016$ and $\mathrm{t}(10)=2.507,0.031$, respectively) and the frequency 
Table 1 T-Maze results

\begin{tabular}{llcc}
\hline Group & Index & Target & Opposite \\
\hline PBS (N=12) & Time spent (sec) & $93.2 \pm 28.4^{*}$ & $65.1 \pm 18.9$ \\
& Distance moved (cm) & $566 \pm 120^{*}$ & $426 \pm 133$ \\
& Frequency (count) & $17.9 \pm 4.3$ & $15.7 \pm 6.6$ \\
PBS + HYP $(\mathrm{N}=11)$ & Time spent (sec) & $78.8 \pm 39.7$ & $75.0 \pm 36.4$ \\
& Distance moved (cm) & $466 \pm 238$ & $467 \pm 270$ \\
& Frequency (count) & $15.8 \pm 7.0$ & $14.0 \pm 6.6$ \\
MgT $(\mathrm{N}=11)$ & Time spent (sec) & $115.2 \pm 47.3^{*}$ & $57.7 \pm 25.9$ \\
& Distance moved (cm) & $513 \pm 169^{*}$ & $351 \pm 152$ \\
& Frequency (count) & $14.3 \pm 4.3$ & $11.2 \pm 3.8$ \\
MgT + HYP $(\mathrm{N}=11)$ & Time spent (sec) & $87.4 \pm 49.3$ & $46.7 \pm 24.3$ \\
& Distance moved (cm) & $483 \pm 173^{*}$ & $304 \pm 159$ \\
& Frequency (count) & $17.9 \pm 6.8^{*}$ & $12.7 \pm 6.1$ \\
\hline
\end{tabular}

Values are mean $\pm S D$. In the training period the target compartment was red + reward, and the opposite compartment was yellow. N represents the number of zebrafish. PBS, phosphate-buffered saline; MgT, magnesium L-threonate; HYP, hypoxia. * $p<0.05$ compared to the opposite compartment

of entries was unchanged ( $\mathrm{t}(10)=1.894, \mathrm{p}=0.088$ ) (Table 1). The MgT + HYP group showed significantly higher distance and frequency of entries in the target compartment compared to the opposite compartment $(\mathrm{t}(10)=2.641, \mathrm{p}=0.025$ and $(\mathrm{t}(10)=3.373, \mathrm{p}=0.007$, respectively), and the difference in time was not significant $(\mathrm{t}(10)=2.140, \mathrm{p}=0.058)($ Table 1$)$.

Figure 5 shows the target compartment preference for time spent, distance moved, and frequency of entries. For all measures, the PBS + HYP group showed no compartment preference.

\section{TTC staining}

Visualization with a microscope showed that the infarct area was reduced in the MgT $+\mathrm{HYP}$ group compared to the PBS + HYP group (Fig. 6a, Additional file 1). In addition, the PBS + HYP group showed significantly lower absorbance than the other groups $(\mathrm{F}(4,30)=24.34$, $\mathrm{p}<0.001$, adjusted $\mathrm{p}=0.001$ for $\mathrm{PBS}+\mathrm{HYP}$ vs PBS, $<0.001$ for PBS+HYP vs MgT and 0.002 for $\mathrm{PBS}+\mathrm{HYP}$ vs MgT $+\mathrm{HYP})$. There was no significant difference in absorbance between the PBS, MgT, and MgT + HYP groups (Fig. 6b).

\section{Western blot}

Western blot revealed upregulation of EAAT4 in the MgT group. Compared to the PBS group, an 18\% decrease was observed in the PBS + HYP group, a 110\% increase was found in the MgT group, and a 35\% increase was present in the MgT + HYP group (this was a $65 \%$ increase

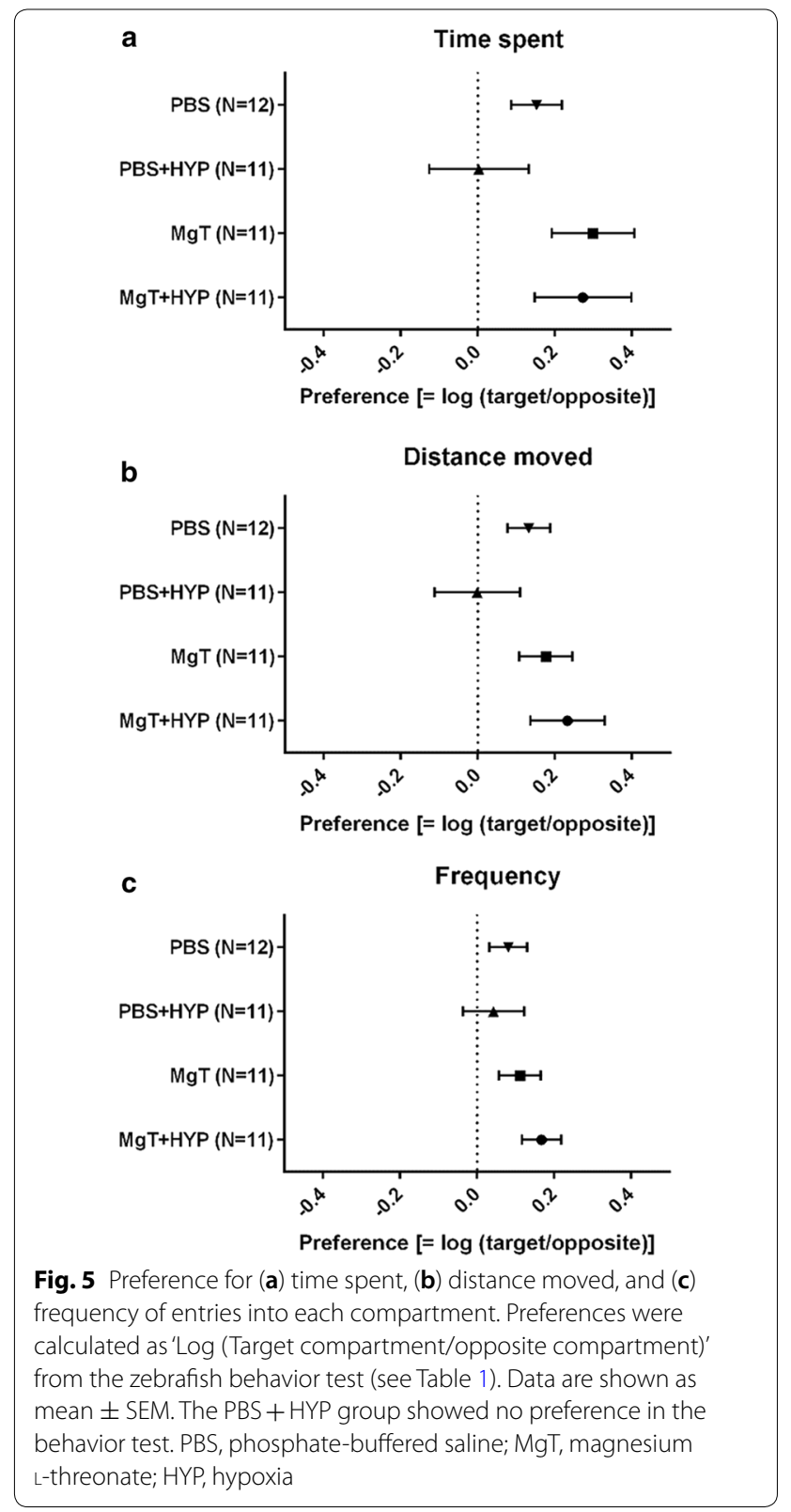

compared to the PBS + HYP group and a 36\% decrease compared to the MgT group) (Fig. 7, Additional file 2).

\section{Discussion}

In this study, we showed MgT may prevent hypoxiainduced cognitive dysfunction with decreased brain infarction and upregulated glutamate transporter (EAAT4). In addition to other studies which showed neuroprotective effects of MgT [12-16], our experiment suggested a new mechanism of $\mathrm{MgT}$ using a novel hypoxic zebrafish model. 


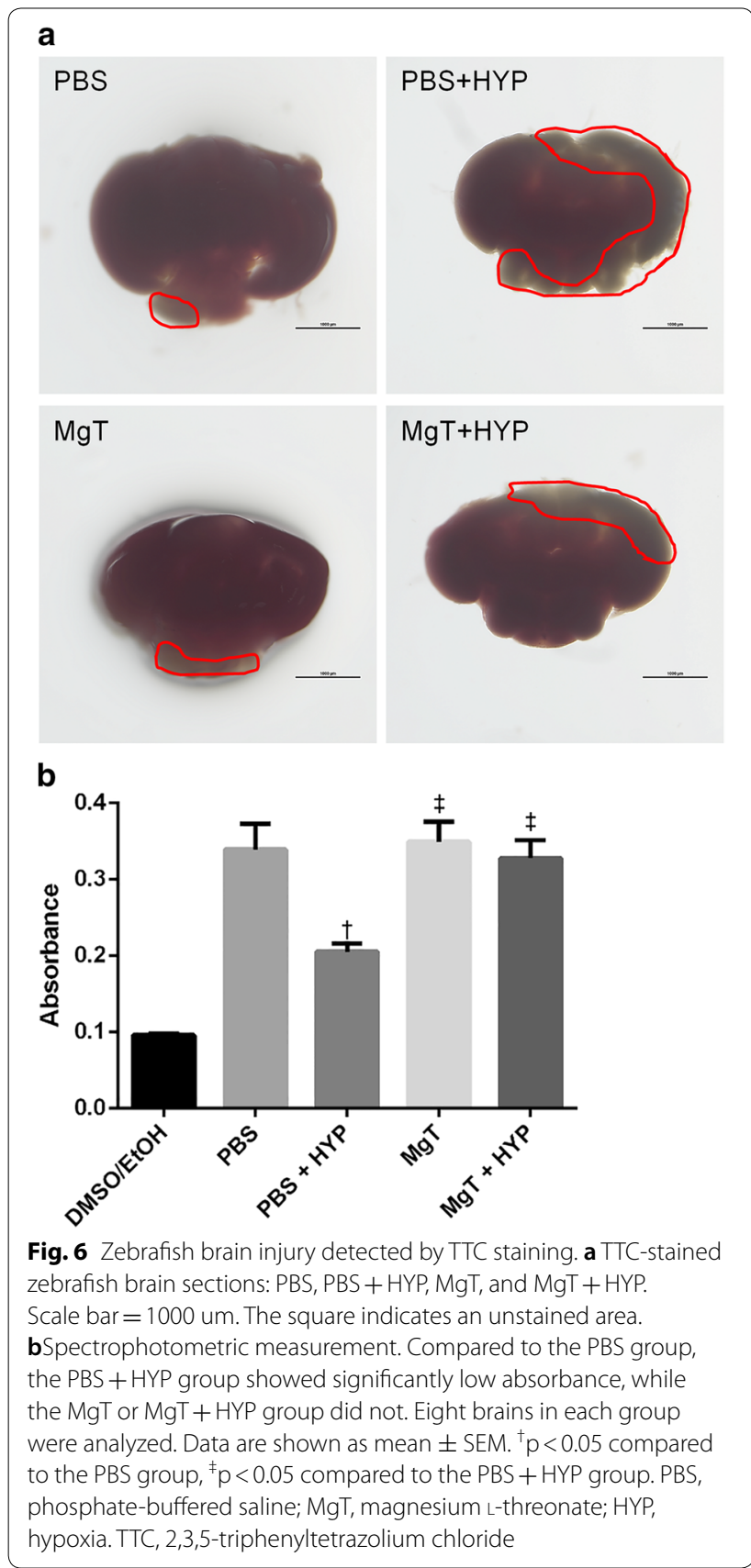

Cerebral hypoxia-ischemia models have been widely used to evaluate mechanisms of neuroprotection [4]. The pathophysiology underlying hypoxic brain damage includes complex mechanisms including loss of ATP, excitotoxicity, production of free radicals, inflammation, over activation of the immune system, and cell death [28]. Although numerous mammalian studies have extensively investigated ischemic stroke, most have failed to develop therapeutic treatments for ischemia. Therefore,
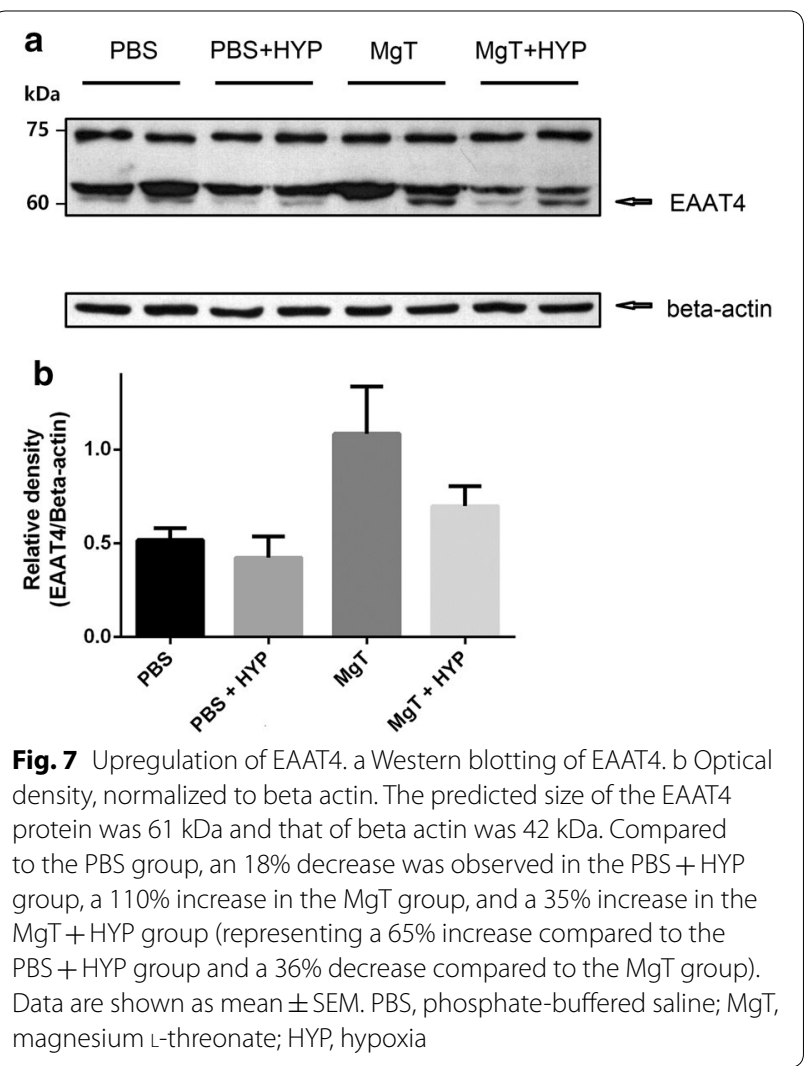

some progress may be made by studying hypoxic-tolerant organisms such as a fish [28].

The zebrafish is a relatively small and simple vertebrate organism, who's genetic composition is similar to that of mammals, including humans. Thus, similar genes are likely to be associated with similar functions in humans [17]. Braga et al. reported spontaneous behavioral recovery in zebrafish after hypoxia [24]. In addition, several zebrafish models have been developed to demonstrate the usefulness of assessing cognitive function, learning, and memory [27, 29]. The zebrafish T-maze is based on visual discrimination learning [30]. Sison and Gerlai evaluated associative memory using visual perception in zebrafish [31]. Notably, zebrafish have specific color preferences [32]. We previously employed a similar zebrafish behavior model using color preferences in the absence of food rewards $[21,26]$. Similar to our previous findings, a four-day training period was sufficient to allow zebrafish to develop a preference for a particular target compartment, even in the absence of colored cellophane and food during the testing period. Approximately $10 \mathrm{~min}$ of hypoxia reversed the effects of training and induced cerebral injury, consistent with our previous results $[21,26]$.

Magnesium is important for proper functioning in many tissues and organs including those of the cardiovascular, neuromuscular, and nervous systems. It plays an 
important role in synaptic plasticity [33] by reducing the calcium dependent post-burst after hyperpolarization of membrane potential, and regulating voltage-dependent blockade of N-methyl-D-aspartate (NMDA) glutamate receptors [23, 34]. Previous studies have shown that an increase in magnesium ion concentration in the extracellular fluid causes long-term enhancement of synaptic plasticity in hippocampal neurons [35]. Thus, increased magnesium in the brain may improve cognitive function.

Nonetheless, McKee et al. [11] reported limited neuroprotective effects of $\mathrm{MgSO}_{4}$ in patients with acute brain injury. Intravenous $\mathrm{MgSO}_{4}$ administration appeared to be hindered by the blood-brain barrier, leading to low levels of magnesium in the CSF, reflective of brain bioavailability $[11,36]$. Compared with $\mathrm{MgSO}_{4}, \mathrm{MgT}$ has a different molecular structure that consists of a magnesium ion and threonate. Interestingly, Slutsky et al. [13] showed that sodium-L-threonate with/without magnesium chloride did not affect memory, while MgT enhanced memory recall. Sun et al. [12] reported several threonate effects, including increased mitochondrial function, glutamatergic synapse density, and neuronal intracellular magnesium ions in hippocampal neuronal cultures. They suggested that threonate may induce magnesium ion transport into hippocampal neurons.

Although reliable increases in magnesium levels are mostly safe [13], magnesium overdose may result in adverse events including lowered blood pressure, slowed heart rate, and cardiac arrhythmia or arrest. Because there was no prior study regarding $\mathrm{MgT}$ concentration, we first evaluated MgT toxicity in zebrafish embryos. After confirming that an MgT concentration below $25 \mathrm{mM}$ showed no adverse effect on development or survival, we then performed a cellular experiment. In aerobic conditions, $\mathrm{MgT}$ and $\mathrm{MgSO}_{4}$ did not alter viability of human neuronal cell cultures. However, in hypoxic conditions, $\mathrm{MgT}$ treatment showed significantly improved cell viability, while $\mathrm{MgSO}_{4}$ treatment was not significant. In addition, the concentration $(1 \mathrm{mM}$ or $10 \mathrm{mM})$ of either $\mathrm{MgT}$ or $\mathrm{MgSO}_{4}$, did not affect outcome. This finding suggests that the regulation of the magnesium occurs at a cellular level at a relatively wide concentration range. We then performed an in vivo experiment with MgT. As expected, zebrafish pretreated with $\mathrm{MgT}$ maintained a preference in time, distance, and frequency of entries to the target compartment after hypoxic insult. The absorbance of zebrafish brain after TTC staining in the MgT + HYP groups was significantly higher than those in the PBS + HYP group. This indicates that MgT preconditioning reduced brain infarction and protected against hypoxic insult, in agreement with the behavioral results.

During hypoxia, many changes, including glutamate alteration, NMDA receptor stimulation, and neuronal degeneration occurred [37]. There are several explanations for neuroprotective effects of magnesium. Stevenson et al. [10] suggested that magnesium protects the central nervous system from hypoxic injuries through the prolyl hydroxylase or factor inhibiting hypoxia-inducible factor pathways. They focused on a specific genetic pathway (ephrinB2a with a hypoxia-inducible transcription factor 1 pathway) in neurodevelopment of zebrafish embryos. Others focused on tumor necrosis factor alpha to explain the neuroprotective effect of magnesium [14, 15]. Other plausible explanations include stabilization of the cell membrane, maintenance of ionic homeostasis by attenuating reductions in $\mathrm{Na}^{+} \mathrm{K}$-ATPase activity, neuronal effects via reduction in NMDA receptor-mediated calcium entry into the cell, and vascular effects by improving cerebral blood flow $[11,37]$.

In this study, we investigated the role of EAAT4 as a neuroprotective mechanism of magnesium. Once released into the synapse, glutamate is rapidly cleared by transporters (high-affinity EAATs) to limit excitotoxicity [38]. Keeping a low concentration of extracellular glutamate is also required for high signal-to-noise ratios during synaptic transmission [6]. Among the glutamate transporters, EAAT4 is expressed in several sites, including the cerebellum, hippocampus, and spinal cord [39-41]. Compared to EAAT subtypes 1-3, EAAT4 is associated with higher chloride conductance, which is not coupled to glutamate uptake, and therefore acts as an inhibitory glutamate receptor $[39,42]$. By switching glutamate transport and chloride channel activity, EAAT4 may dampen cellular excitability during glutamate uptake and prevent a reduction in transport rate [42]. A previous study found that Purkinje cells die more easily in the event of EAAT4 deficiency after global brain ischemia [43]. Yi et al. [40] reported upregulated EAAT4 in rat hippocampal astrocytes 3-7 days after traumatic brain injury. In addition, Sachs et al. [44] suggested a role of EAAT4 in neuroprotection using a mutant neurodegenerative rat model. These findings suggest that EAAT4 may play a role, although the neuroprotective mechanisms are complex [45]. In this study, MgT groups showed upregulation of EAAT4 protein, which is approximately $61 \mathrm{kDa}$ in size. Based on our results, MgT upregulates EAAT4 by $65-110 \%$, whereas hypoxia downregulates EAAT4 by $18-36 \%$. Taken together with previous experimental results, hypoxia may initially deplete EAAT4 and then induce EAAT4 reactivity in a later recovery phase [40]. Upregulation of EAAT4 by MgT appeared to protect the brain from hypoxic injury. A schematic diagram of the proposed mechanism of hypoxia and magnesium interaction is described in Fig. 8.

Western blotting demonstrated two unidentified protein bands expressed consistently in all groups. 


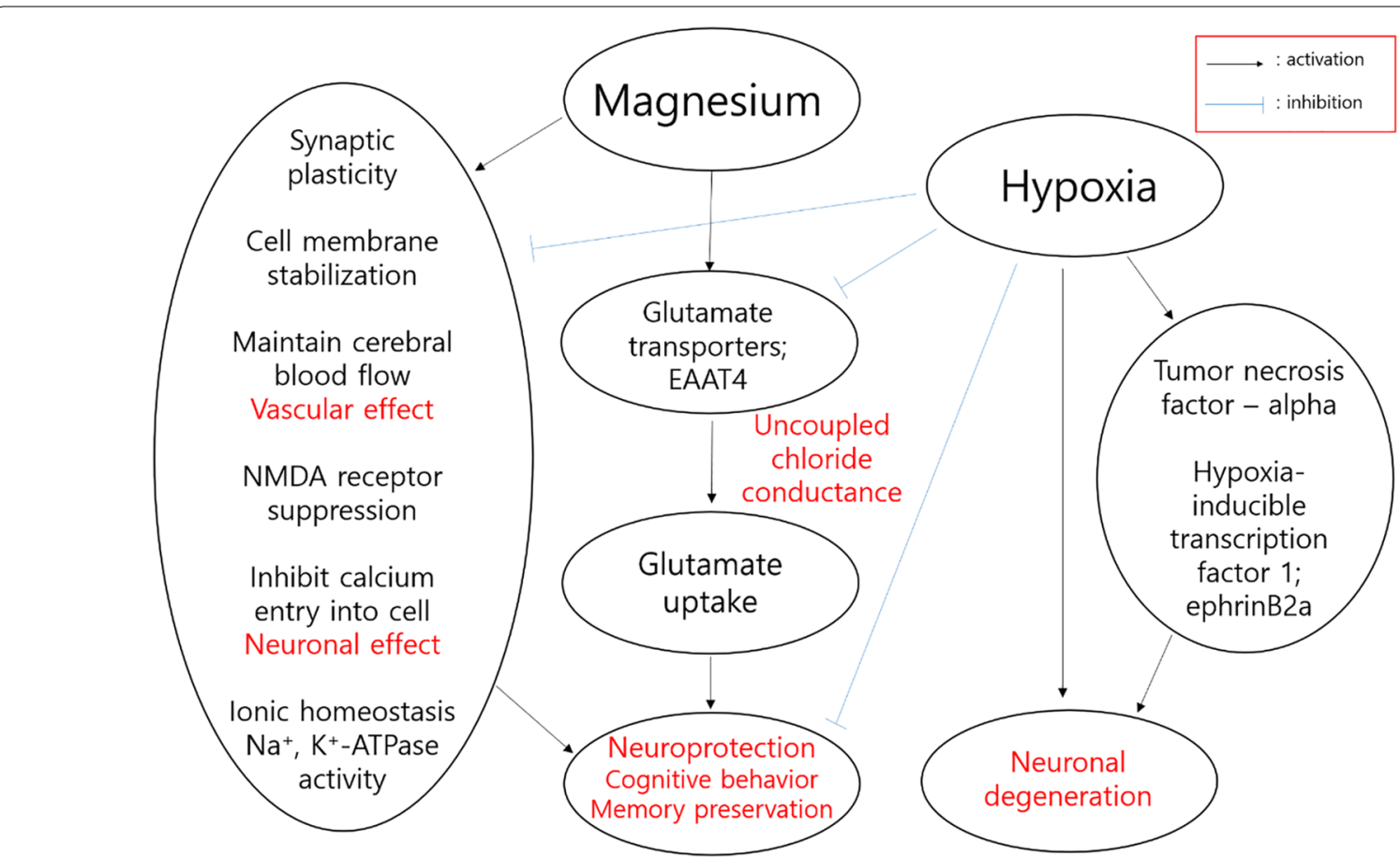

Fig. 8 Schematic of the proposed mechanisms of hypoxia and magnesium. Black lines with arrows indicate activation while blue lines indicate inhibition. The red-colored texts represented the key concepts in the schematic diagram

A consignment test also showed the two additional unknown bands mentioned above (data not shown). We referred to the antibody manufacturer's guidelines to verify whether these bands may represent non-specific binding, or be due to incomplete antibody validation in the zebrafish. The antibody used was rabbit-derived, and validated in mice. One of the limitations of our study was that there was no suitable commercial antibody for zebrafish, making further evaluation of the mechanism limited. Moreover, it would be interesting to confirm the expression of EAAT4 several days following hypoxia. Another limitation of this study was that we performed tricaine anesthesia for oral administration of MgT or PBS prior to hypoxia and behavior testing. Although time under anesthesia was minimal, it may have influenced the outcomes via anesthetic preconditioning or toxic effects.

Despite these limitations, this study has several advantages. To the best of our knowledge, this study is the first application of drug-induced memory preservation in the hypoxic zebrafish behavior model. Because zebrafish are inexpensive and easy to manage, using our protocol may be useful to apply similar experiments designed to confirm the protective or toxic effects of other drugs. This finding suggests that hypoxic-tolerant organisms appear to have adaptive mechanisms to overcome hypoxic damage. Further experiments and observations are required to evaluate the specific mechanisms in order to contribute to the clinical application of potential treatments.

\section{Conclusion}

This study showed that pretreatment with MgT, and subsequent upregulation of glutamate transporter EAAT4, has protective effects on neuronal survival, reduction in cerebral infarction, and preservation of learning and memory in zebrafish following hypoxia.

\section{Supplementary information}

Supplementary information accompanies this paper at https://doi. org/10.1186/s12868-020-00580-6.

Additional file 1: Figure S1. Original version of TTC-stained zebrafish brain sections: PBS, PBS + HYP, MgT, and MgT + HYP. Scale bar $=1000$ um (See Figure 6a).

Additional file 2: Figure S2. Original version of western blotting of EAAT4 (See Figure 7a).

\section{Abbreviations}

CSF: Cerebrospinal fluid; DMSO: Dimethyl sulfoxide; DO: Dissolved oxygen; EAAT: Excitatory amino acid transporter; MgSO4: Magnesium sulfate; MgT: Magnesium L-threonate; PBS: Phosphate-buffered saline; TBS: Tris-buffered saline; TTC: 2,3,5-triphenyltetrazolium chloride. 


\section{Acknowledgements \\ Not applicable.}

\section{Authors' contributions}

YSK and IOL conceived the study, conceptualized the experimental design YHK and TJM gave input to experimental design. YSK and YHK performed the imaging and clinical assessments and acquired the data. YSK, TJM and YHK recruited the subjects. YJW, BGL and IOL organized the study. YSK wrote the first draft and prepared the manuscript. YSK and YHK generated the figures. YJW, BGL and IOL analyzed the data. YSK, YJW, BGL, TJM and IOL interpreted the data. All authors revised and gave input to the manuscript. All authors read and approved the final manuscript.

\section{Authors information}

First author, Young Sung Kim, M.D., Ph.D. Clinical Assistant Professor, Department of Anesthesiology and Pain Medicine, Korea University Guro Hospital, Seoul, Korea; Corresponding author, II ok Lee, M.D., Ph.D. Professor, Department of Anesthesiology and Pain Medicine, Korea University Guro Hospital, Seoul, Korea.

\section{Funding}

This research did not receive any specific grant from funding agencies in the public, commercial, or not-for-profit sectors.

\section{Availability of data and materials}

Data are available from Pf. II-Ok Lee, Department of Anesthesiology and Pain Medicine, Korea University Guro Hospital, Seoul, Korea; E-mail: iloklee@korea. ac.kr for researchers who meet the criteria for access to confidential data.

\section{Ethics approval and consent to participate}

The study was approved by the Committee on the Ethics of Animal Experiments of the Korea University Medical School. (IACUC number KOREA-2018-0032).

\section{Consent for publication}

$$
\text { Not applicable. }
$$

\section{Competing interests}

The authors declare that they have no competing interests.

\section{Author details}

${ }^{1}$ Department of Anesthesiology and Pain Medicine, Korea University Guro Hospital, Seoul, Korea. ${ }^{2}$ Department of Anesthesiology and Pain Medicine, Korea University Ansan Hospital, Ansan, Korea. ${ }^{3}$ Institute of Medical Science, Korea University Ansan Hospital, Korea University College of Medicine, Ansan, Korea.

Received: 26 November 2019 Accepted: 21 June 2020

Published online: 26 June 2020

\section{References}

1. Min TJ, Kim WY, Kim YS, Yoo JW, Lee HJ, Lee IO. Ulinastatin up-regulates glutamate transporters in glial cells. Int J Clin Exp Med. 2016;9(2):2367-74.

2. McLean SL, Grayson B, Marsh S, Zarroug SH, Harte MK, Neill JC. Nicotinic alpha7 and alpha4beta2 agonists enhance the formation and retrieval of recognition memory: potential mechanisms for cognitive performance enhancement in neurological and psychiatric disorders. Behav Brain Res. 2016;302:73-80

3. Frati P, Kyriakou C, Del Rio A, Marinelli E, Vergallo GM, Zaami S, et al. Smart drugs and synthetic androgens for cognitive and physical enhancement: revolving doors of cosmetic neurology. Curr Neuropharmacol. 2015;13(1):5-11.

4. Mandel M, Talamoni FonoffE, Bor-Seng-Shu E, Teixeira MJ, Chadi G. Neurogenic neuroprotection: clinical perspectives. Funct Neurol. 2012;27(4):207-16.

5. Oechmichen M, Meissner C. Cerebral hypoxia and ischemia: the forensic point of view: a review. J Forensic Sci. 2006;51(4):880-7.

6. Danbolt NC. Glutamate uptake. Prog Neurobiol. 2001;65(1):1-105.
7. Gibbs ME, Hertz L, Ng KT. Inhibition of short-term memory formation in the chick by blockade of extracellular glutamate uptake. Neurobiol Learn Mem. 2004;81(2):115-9.

8. Bickler PE, Fahlman CS, Ferriero DM. Hypoxia increases calcium flux through cortical neuron glutamate receptors via protein kinase C. J Neurochem. 2004;88(4):878-84.

9. Inage YW, Itoh M, Wada K, Takashima S. Expression of two glutamate transporters, GLAST and EAAT4, in the human cerebellum: their correlation in development and neonatal hypoxic-ischemic damage. J Neuropathol Exp Neurol. 1998;57(6):554-62.

10. Stevenson TJ, Trinh T, Kogelschatz C, Fujimoto E, Lush ME, Piotrowski T, et al. Hypoxia disruption of vertebrate CNS pathfinding through ephrinB2 is rescued by magnesium. PLoS Genet. 2012;8(4):e1002638.

11. McKee JA, Brewer RP, Macy GE, Borel CO, Reynolds JD, Warner DS. Magnesium neuroprotection is limited in humans with acute brain injury. Neurocrit Care. 2005;2(3):342-51.

12. Sun $Q$, Weinger JG, Mao F, Liu G. Regulation of structural and functional synapse density by L-threonate through modulation of intraneuronal magnesium concentration. Neuropharmacology. 2016;108:426-39.

13. Slutsky l, Abumaria N, Wu LJ, Huang C, Zhang L, Li B, et al. Enhancement of learning and memory by elevating brain magnesium. Neuron. 2010;65(2):165-77.

14. Wang J, Liu Y, Zhou LJ, Wu Y, Li F, Shen KF, et al. Magnesium L-threonate prevents and restores memory deficits associated with neuropathic pain by inhibition of TNF-alpha. Pain Physician. 2013;16(5):E563-75.

15. Xu T, Li D, Zhou X, Ouyang HD, Zhou $\sqcup$, Zhou H, et al. Oral application of magnesium-L-threonate attenuates vincristine-induced allodynia and hyperalgesia by normalization of tumor necrosis factor-alpha/nuclear factor-kappaB signaling. Anesthesiology. 2017;126(6):1151-68.

16. Li W, Yu J, Liu Y, Huang XJ, Abumaria N, Zhu Y, et al. Elevation of brain magnesium prevents synaptic loss and reverses cognitive deficits in Alzheimer's disease mouse model. Mol Brain. 2014;7:65.

17. Howe K, Clark MD, Torroja CF, Torrance J, Berthelot C, Muffato M, et al. The zebrafish reference genome sequence and its relationship to the human genome. Nature. 2013;496(7446):498-503.

18. Kalueff AV, Stewart AM, Gerlai R. Zebrafish as an emerging model for studying complex brain disorders. Trends Pharmacol Sci. 2014;35(2):63-75.

19. Shams S, Rihel J, Ortiz JG, Gerlai R. The zebrafish as a promising tool for modeling human brain disorders: a review based upon an IBNS Symposium. Neurosci Biobehav Rev. 2018;85:176-90.

20. Collier AD, Khan KM, Caramillo EM, Mohn RS, Echevarria DJ. Zebrafish and conditioned place preference: a translational model of drug reward. Prog Neuropsychopharmacol Biol Psychiatry. 2014;55:16-25.

21. Kim YH, Lee KS, Park AR, Min TJ. Adding preferred color to a conventional reward method improves the memory of zebrafish in the T-maze behavior model. Anim Cells Syst. 2017;21(6):374-81.

22. Westermaier T, Stetter C, Kunze E, Willner N, Raslan F, Vince GH, et al. Magnesium treatment for neuroprotection in ischemic diseases of the brain. Exp Transl Stroke Med. 2013;5(1):6

23. Do SH. Magnesium: a versatile drug for anesthesiologists. Korean J Anesthesiol. 2013;65(1):4-8.

24. Braga MM, Rico EP, Cordova SD, Pinto CB, Blaser RE, Dias RD, et al. Evaluation of spontaneous recovery of behavioral and brain injury profiles in zebrafish after hypoxia. Behav Brain Res. 2013;253:145-51.

25. Kulkarni P, Chaudhari GH, Sripuram V, Banote RK, Kirla KT, Sultana R, et al. Oral dosing in adult zebrafish: proof-of-concept using pharmacokinetics and pharmacological evaluation of carbamazepine. Pharmacol Rep. 2014;66(1):179-83.

26. Kim YH, Lee KS, Kim YS, Kim YH, Kim JH. Effects of hypoxic preconditioning on memory evaluated using the T-maze behavior test. Anim Cells Syst. 2019;23(1):10-7.

27. Yu X, Li YV. Zebrafish as an alternative model for hypoxic-ischemic brain damage. Int J Physiol Pathophysiol Pharmacol. 2011:3(2):88-96.

28. Bickler PE. Clinical perspectives: neuroprotection lessons from hypoxiatolerant organisms. J Exp Biol. 2004:207(Pt 18):3243-9.

29. Levin ED, Chen E. Nicotinic involvement in memory function in zebrafish. Neurotoxicol Teratol. 2004:26(6):731-5.

30. Colwill RM, Raymond MP, Ferreira L, Escudero H. Visual discrimination learning in zebrafish (Danio rerio). Behav Processes. 2005;70(1):19-31. 
31. Sison M, Gerlai R. Associative learning in zebrafish (Danio rerio) in the plus maze. Behav Brain Res. 2010;207(1):99-104.

32. Avdesh A, Martin-Iverson MT, Mondal A, Chen M, Askraba S, Morgan N, et al. Evaluation of color preference in zebrafish for learning and memory. J Alzheimers Dis. 2012;28(2):459-69.

33. Xu ZP, Li L, Bao J, Wang ZH, Zeng J, Liu EJ, et al. Magnesium protects cognitive functions and synaptic plasticity in streptozotocin-induced sporadic Alzheimer's model. PLoS ONE. 2014;9(9):e108645.

34. Glasdam SM, Glasdam S, Peters GH. The importance of magnesium in the human body: a systematic literature review. Adv Clin Chem. 2016;73:169-93.

35. Slutsky I, Sadeghpour S, Li B, Liu GS. Enhancement of synaptic plasticity through chronically reduced $\mathrm{Ca} 2+$ flux during uncorrelated activity. Neuron. 2004:44(5):835-49.

36. Brewer RP, Parra A, Borel CO, Hopkins MB, Reynolds JD. Intravenous magnesium sulfate does not increase ventricular CSF ionized magnesium concentration of patients with intracranial hypertension. Clin Neuropharmacol. 2001;24(6):341-5.

37. Hoffman DJ, Marro PJ, McGowan JE, Mishra OP, Delivoria-Papadopoulos M. Protective effect of MgSO4 infusion on nmda receptor binding characteristics during cerebral cortical hypoxia in the newborn piglet. Brain Res. 1994;644(1):144-9.

38. Santello M, Volterra A. Synaptic modulation by astrocytes via Ca2+ -dependent glutamate release. Neuroscience. 2009;158(1):253-9.

39. Dalet A, Bonsacquet J, Gaboyard-Niay S, Calin-Jageman I, Chidavaenzi RL, Venteo $\mathrm{S}$, et al. Glutamate transporters EAAT4 and EAAT5 are expressed in vestibular hair cells and calyx endings. PLoS ONE. 2012;7(9):e46261.
40. $\mathrm{Yi} \mathrm{JH}$, Herrero R, Chen G, Hazell AS. Glutamate transporter EAAT4 is increased in hippocampal astrocytes following lateral fluid-percussion injury in the rat. Brain Res. 2007;1154:200-5.

41. Hu WH, Walters WM, Xia XM, Karmally SA, Bethea JR. Neuronal glutamate transporter EAAT4 is expressed in astrocytes. Glia. 2003;44(1):13-25.

42. Mim C, Balani P, Rauen T, Grewer C. The glutamate transporter subtypes EAAT4 and EAATs 1-3 transport glutamate with dramatically different kinetics and voltage dependence but share a common uptake mechanism. J Gen Physiol. 2005;126(6):571-89.

43. Welsh JP, Yuen G, Placantonakis DG, Vu TQ, Haiss F, O'Hearn E, et al. Why do Purkinje cells die so easily after global brain ischemia? Aldolase C, EAAT4, and the cerebellar contribution to posthypoxic myoclonus. Adv Neurol. 2002;89:331-59.

44. Sachs AJ, David SA, Haider NB, Nystuen AM. Patterned neuroprotection in the Inpp4a(wbl) mutant mouse cerebellum correlates with the expression of EAAT4. PLOS ONE. 2009;4(12):e8270.

45. Vazana U, Veksler R, Pell GS, Prager O, Fassler M, Chassidim Y, et al. Glutamate-mediated blood-brain barrier opening: implications for neuroprotection and drug delivery. J Neurosci. 2016;36(29):7727-39.

\section{Publisher's Note}

Springer Nature remains neutral with regard to jurisdictional claims in published maps and institutional affiliations.
Ready to submit your research? Choose BMC and benefit from:

- fast, convenient online submission

- thorough peer review by experienced researchers in your field

- rapid publication on acceptance

- support for research data, including large and complex data types

- gold Open Access which fosters wider collaboration and increased citations

- maximum visibility for your research: over $100 \mathrm{M}$ website views per year

At BMC, research is always in progress.

Learn more biomedcentral.com/submissions 